\title{
Comportamento colorimétrico da madeira de maçaranduba tratada com produtos de acabamento
}

\author{
Juliana Holanda MAIA ${ }^{1 *}$, Marília Graziela Dantas MOURA ${ }^{1}$, Arthur Santos MAGALHÃES ${ }^{1}$, \\ Vinícius Gomes de CASTRO ${ }^{1}$
}

${ }^{1}$ Departamento de Ciências Agronômicas e Florestais, Universidade Federal do Semi-Árido, Mossoró, RN, Brasil.

*E-mail: julianahmaia23@gmail.com

Recebido em janeiro/2018; Aceito em junho/2018.

\begin{abstract}
RESUMO: O comércio da madeira de maçaranduba (Manilkara sp.) é muito popular na região Nordeste do Brasil devido suas características físico-mecânicas e de sua aparência estética. O objetivo do presente trabalho foi analisar a cor da madeira de maçaranduba comercializada em diferentes estados nordestinos, assim como o efeito de quatro diferentes produtos de acabamento (verniz poliuretano; cera de carnaúba industrializada e bruta; e óleo de linhaça). Os corpos de prova foram submetidos ao intemperismo natural por quatro meses e tiveram a alteração da cor avaliada pelo sistema CIELa*b*. Não houve variação nos parâmetros colorimétricos das madeiras comercializadas em diferentes localidades. O óleo de linhaça foi o produto que apresentou maior capacidade de mudança na cor da madeira, evidenciando a tonalidade avermelhada. Porém foi o uso do verniz que apresentou uma cor mais estável após os quatro meses de envelhecimento.
\end{abstract}

Palavras-chave: CIELa*b*, verniz, cera de Carnaúba, óleo de linhaça.

\section{Color behavior of massaranduba wood treated with finishing products}

\begin{abstract}
Massaranduba (Manilkara sp.) wood trade is very popular in the Brazilian Northeast region due its physico-mechanical properties and esthetical appearance. The aim of this work was evaluate the Massaranduba wood color traded in different Northeast states, as analyze the effect of four different finishing product (polyurethane varnish; Industrialized and raw Carnauba wax; and linseed oil). Wood specimens were submitted to outdoor weathering for four months and the color change was measure according to the CIELa*b*system. There was no colorimetric parameters variation among wood traded in different locations. Linseed oil was the finishing product with higher capacity of color change, highlighting the red ton of the Massaranduba. However, the use of varnish showed a more stable color after the four months of aging.

Keywords: CIELa*b*, varnish, Carnauba wax, linseed oil.
\end{abstract}

\section{INTRODUÇÃO}

As espécies do gênero Manilkara, popularmente conhecidas como maçarandubas, são espécies de alta densidade $\left(1 \mathrm{~g} \mathrm{~cm}^{-3}\right)$ e resistência estrutural (classe C60) (ROSA et al., 2014). A Manilkara sp. é uma das espécies madeireiras amazônicas mais exploradas e comercializadas. Por exemplo, no município de Portel-PA, dos $343.643,83 \mathrm{~m}^{3}$ de madeira legalmente transportada no ano de $2009,13,55 \%$ foram referentes à madeira de maçaranduba (SANTANA et al., 2010).

Os estados da Região Nordeste são grandes consumidores deste tipo de madeira, principalmente voltada para construção civil e indústria de móveis (SANTANA et al., 2010). Nestes mercados, a cor e aparência estética do produto tem tanta importância quanto as propriedades mecânicas da madeira. Fiedler et al. (2012) caracterizaram as madeiras serradas de três municípios do Pará e observaram que $49 \%$ das espécies utilizadas são classificadas como do grupo "vermelhas", do qual a maçaranduba é o principal representante.

Porém, a cor da madeira não é uma característica perene, ela pode ser alterada pela umidade, temperatura, ou por deteriorações biológicas (ATAYDE et al., 2011). Desta forma, o conhecimento não só da cor, mas também de sua variação em função de produtos de acabamento aplicados na madeira e do tempo da sua exposição à intempérie torna-se fundamental para o uso a comercialização do produto (QUEIROZ et al, 2016).

A madeira exposta à radiação solar está sujeita à degradação de sua superfície inicialmente com a mudança de cor e seguido com a perda de suas propriedades mecânicas. E, embora o uso de produtos de acabamento possa alterar a cor natural da madeira, também pode conservar a cor original por períodos maiores de exposição (GONÇALEZ et al, 2010). Segundo Baysal et al. (2013) o uso de produtos de acabamento também protege a dureza da madeira, que é afetada pela despolimerização da lignina e de hidratos de carbonos que compõem a parede celular em exposições prolongadas à luz solar.

Santos; Duarte (2013) afirmam ainda que a proteção superficial impede a entrada de água na madeira e, consequentemente, a deposição de agentes deterioradores como fungos que podem vir a alterar a cor. Estes produtos de acabamentos podem ser origem natural ou sintética (SOUZA et al., 2011). Os produtos sintéticos mais utilizados na indústria moveleira são as tintas, os vernizes e seladoras. As ceras e os óleos são produtos de origem natural tendo como função proteger, limpar, lustrar e conservar. A cera de carnaúba é um exemplo deste tipo de produto. Dos óleos, o mais conhecido e mais usado na pintura industrial é o óleo de linhaça (SANTOS; DUARTE, 2013). 
O óleo de linhaça é um produto rico em ácidos graxos não saturados como os ácidos linoleicos. Através da polimerização destes ácidos pela oxidação, a película de óleo aplicada na madeira tende a endurecer (CHANG; LU, 2013). Já a cera de carnaúba é formada principalmente por ésteres graxos e cadeias longas de alcoóis, ácidos e hidrocarbonetos. Os componentes lipofílicos da cera são capazes de formar uma película que protege e repele a água, tornando-a um bom produto para aplicação em móveis e pisos, sem comprometer $\mathrm{o}$ aspecto e estrutura original da madeira (BULIAN; GRAYSTONE, 2009).

A colorimetria é a ciência que descreve cada elemento da composição de uma cor, numericamente, por meio de aparelhos que avaliam a interação provocada pela luz em uma superfície. Esta forma de atribuição da coloração de uma madeira é importante, pois a simples análise visual pode vir a ser subjetiva (SILVA et al, 2015). O sistema mais utilizado é o CIE L a*b*, que define a sensação da cor com base em três elementos: luminosidade ("L"), que define a escala de cinza entre o branco e o preto; a matiz expressa pelas cores primárias: vermelho - verde $\left(\mathrm{a}^{*}\right)$ e amarelo - azul $\left(\mathrm{b}^{*}\right)$ e a saturação ("C") (CAMARGOS; GONÇALEZ, 2001).

O objetivo desse estudo foi analisar o efeito da aplicação de quatro diferentes produtos de acabamento (verniz poliuretano, óleo de linhaça, cera de carnaúba industrializada e bruta diluída em querosene) em peças de madeira de Manilkara sp. comercializadas na Região Nordeste do Brasil, comparando-se a cor antes e após envelhecimento natural.

\section{MATERIAL E MÉTODOS}

Peças de madeira comercializadas como maçaranduba (Manilkara sp.) foram obtidas em duas madeireiras da cidade de Fortaleza-CE, quatro madeireiras de Mossoró-RN, uma madeireira de Caraúbas-RN, duas madeireiras de Petrolina-PE e uma madeireira de Valença-BA. Para a avaliação do padrão de cor das peças de diferentes procedências foram produzidos 3 corpos-de-prova das peças de cada madeireira com largura e comprimento médios superiores a $5 \mathrm{~cm}$ (diâmetro do leitor do colorímetro). Os corpos-de-prova foram lixados manualmente seguindo a ordem de lixas de grãs 80,150 e 220, de acordo com metodologia sugerida por Souza et al. (2011). Após o lixamento, foram efetuadas as mensurações de coloração utilizando colorímetro Konica Minolta CR-410 que mensura conforme o sistema CIE-L*a*b*. Foram feitas três leituras para cada corpo-de-prova dos parâmetros colorimétricos: L (luminosidade), $a^{*}$ (coordenada no eixo vermelho-verde) e b* (coordenada do eixo azul-amarelo). Para o cálculo dos parâmetros C (saturação) e $h^{*}($ ângulo de tinta), foram utilizadas as equações (Equação 1) e (Equação 2).

$$
\begin{aligned}
& \mathrm{C}=\left(\mathrm{a}^{*} 2+\mathrm{b}^{*} 2\right) / 2 \\
& \mathrm{~h} *=\tan ^{-1}\left(\mathrm{~b}^{*} / \mathrm{a}^{*}\right)
\end{aligned}
$$

(Equação2)

em que: $\mathrm{C}=$ Saturação; $\mathrm{a}^{*}=$ coordenada no eixo vermelho-verde); $\mathrm{b}^{*}=$ coordenada no eixo azul-amarelo; $\mathrm{h}^{*}=$ ângulo de tinta

\subsection{Aplicação dos produtos de acabamento}

Após verificação da homogeneidade das cores das peças de diferentes procedências, 30 corpos de prova foram selecionados aleatoriamente dentre indivíduos com parâmetros colorimétricos estatisticamente iguais comprovados pelo teste de Tukey a 5\% de probabilidade. Os corpos de prova foram então divididos em cinco tratamentos de acordo com o tipo de produto de acabamento utilizado: verniz poliuretano; cera de carnaúba industrializada; cera de carnaúba bruta diluída em querosene; e óleo de linhaça. Todos os produtos foram adquiridos em lojas especializadas, com exceção da cera de carnaúba bruta, que foi fornecida em forma sólida, sem adição de conservantes, diretamente do produtor.

Os quatro produtos utilizados foram aplicados com três demãos, tendo intervalo de 3 horas entre cada uma. Após a secagem da última camada, as amostras com verniz e cera de carnaúba seguiram para o lixamento manual com lixa de grã 400, para minimizar a rugosidade das películas.

\subsection{Determinação de aderência}

Após secagem do produto de acabamento, a aderência foi determinada pelo método de corte em grade adaptado na norma NBR 11003 (ABNT, 2009). Seis cortes paralelos foram feitos com estilete a uma distância de $2 \mathrm{~mm}$ e, em seguida, mais seis cortes paralelos foram feitos no sentido perpendicular ao primeiro grupo de corte. Estes cortes resultaram em uma grade de 25 quadrados, na qual foi aplicada uma fita adesiva semitransparente. Após pressão inicial, a fita foi arrancada. Com auxílio de uma lupa, a ocorrência de destacamento entre as camadas foi observada e registrada.

\subsection{Envelhecimento natural}

Os corpos-de-prova foram expostos ao ar livre durante cinco meses. A cor foi aferida mensalmente em todas as amostras, sendo estas retornadas a exposição à intempérie após a medição. Para cada amostra foram feitas três leituras dos padrões colorimétricos em pontos aleatórios no plano longitudinal. O experimento foi realizado no município de Mossoró - RN (latitude -5,205933; longitude -37,319585) e teve início no dia 25 de julho (mês 0) de 2017 e as demais medições ocorreram no dia 23 de agosto (mês 1), 25 de setembro (mês 2), 24 de outubro (mês 3), 24 de novembro (mês 4) de 2017.

Os corpos-de-prova foram expostos a um ângulo de $45^{\circ} \mathrm{e}$ orientados no eixo Leste-Oeste, voltado para o Norte, seguindo uma adaptação da norma européia EN 927-3 (EN, 2000) ao intemperismo natural. Durante o período de exposição a temperatura média foi de $28^{\circ} \mathrm{C}$, a umidade relativa média de $55 \%$, a radiação solar incidente média de $251,5 \mathrm{~W} \mathrm{~m}^{-2}$, velocidade média do vendo de $2,5 \mathrm{~m} \mathrm{~s}^{-1}$ e a precipitação total de $0,6 \mathrm{~mm}$ (parâmetros monitorados pela Estação Meteorológica Automática da Universidade Federal Rural do Semi-árido). Para mesurar as alterações na cor, foi utilizada a (Equação 3):

$$
\Delta \mathrm{E}=\sqrt{\Delta L^{2}+\Delta a^{2}+\Delta b^{2}} \quad \text { (Equação 3) }
$$

em que: $\Delta \mathrm{E}=$ variação da cor; $\Delta \mathrm{L}=$ variação da luminosidade; $\Delta \mathrm{a}=$ variação da coordenada no eixo vermelho-verde; $\Delta b=$ variação da coordenada no eixo azul-amarelo.

Sendo que se foi utilizado a média dos parâmetros colorimétricos dos tratamentos antes e após a aplicação dos produtos de acabamento para se calcular a variação na cor. Foi realizada análise de variância para avaliar o efeito do local de origem de comercialização da madeira e do intemperismo sobre os parâmetros colorimétricos. Nos casos de resultados significativos, testes de diferença de médias Tukey foram aplicados a $5 \%$ de probabilidade de erro. 


\section{RESULTADOS}

3.1. Variação colorimétrica da madeira de maçaranduba comercializada em diferentes cidades nordestinas

A Tabela 1 apresenta os valores médios dos parâmetros colorimétricos medidos em peças de madeira de maçaranduba comercializadas nos estados da Bahia (BA), Ceará (CE), Pernambuco (PE) e Rio Grande do Norte (RN).

Não houve diferença estatística entre os parâmetros colorimétricos de peças de Maçaranduba comercializadas em diferentes estados da Região Nordeste.

Tabela 1. Parâmetros colorimétricos de peças de maçaranduba comercializadas em diferentes estados do nordeste brasileiro.

Table 1. Colorimetric parameters of Massaranduba wood traded on different Brazilian northeast states.

\begin{tabular}{ccccc}
\hline & \multicolumn{4}{c}{ Estado de origem da madeira } \\
\cline { 2 - 5 } & BA & CE & PE & RN \\
\hline $\mathrm{L}^{*}$ & $40,9^{\mathrm{ns}}$ & $41,19^{\mathrm{ns}}$ & $42,19^{\mathrm{ns}}$ & $41,93^{\mathrm{ns}}$ \\
& $(0,66)$ & $(5,80)$ & $(9,55)$ & $(10,16)$ \\
$\mathrm{a}^{*}$ & $12,45^{\mathrm{ns}}$ & $11,21^{\mathrm{ns}}$ & $11,19^{\mathrm{ns}}$ & $11,79^{\mathrm{ns}}$ \\
& $(0,16)$ & $(18,13)$ & $(8,53)$ & $(9,06)$ \\
$\mathrm{b}^{*}$ & $11,75^{\mathrm{ns}}$ & $11,63^{\mathrm{ns}}$ & $14,85^{\mathrm{ns}}$ & $14,57^{\mathrm{ns}}$ \\
& $(0,86)$ & $(3,11)$ & $(20,75)$ & $(24,36)$ \\
$\mathrm{C}$ & $17,12^{\mathrm{ns}}$ & $16,23^{\mathrm{ns}}$ & $18,63^{\mathrm{ns}}$ & $18,82^{\mathrm{ns}}$ \\
& $(0,49)$ & $(8,48)$ & $(16,13)$ & $(17,45)$ \\
$\mathrm{h}^{*}$ & $46,64^{\mathrm{ns}}$ & $46,47^{\mathrm{ns}}$ & $52,45^{\mathrm{ns}}$ & $50,33^{\mathrm{ns}}$ \\
& $(0,43)$ & $(11,72)$ & $(7,20)$ & $(9,32)$ \\
\hline
\end{tabular}

$\mathrm{ns}=$ diferença não significativa para valores na mesma linha; $\mathrm{L}^{*}=$ luminosidade; $\mathrm{a}^{*}=$ matiz vermelha; $\mathrm{b}^{*}=$ matiz amarela; $\mathrm{C}=$ saturação; $\mathrm{h}^{*}=$ ângulo de tinta; Valores entre parênteses referentes ao coeficiente de variação.

\subsection{Aderência dos produtos de acabamento}

As aderências de todos os produtos foram consideradas satisfatórias (classificação $\mathrm{Gr}_{2}$, de acordo com a norma brasileira). Classificados na sequência de produto de acabamento de menor para maior área de película destacada: verniz (5\%), cera de carnaúba industrializada $(8 \%)$, óleo de linhaça $(12 \%)$ e cera de carnaúba bruta $(13 \%)$.

3.3. Efeito dos produtos de acabamento e envelhecimento natural na cor da madeira

As variações nos parâmetros colorimétricas causados pela aplicação dos produtos de acabamento estudados na madeira de maçaranduba foram apresentadas na Tabela 2.

Tabela 2. Variação nos parâmetros colorimétricos de peças de maçaranduba tratadas com diferentes produtos de acabamento.

Table 2. Variation of colorimetric parameters of Massaranduba wood treated with different finishing products.

\begin{tabular}{lcccc}
\hline \multicolumn{1}{c}{ Acabamento } & $\Delta \mathrm{L}^{*}$ & $\Delta \mathrm{a}^{*}$ & $\Delta \mathrm{b}^{*}$ & $\Delta \mathrm{E}$ \\
\hline Verniz & $-4,45$ & $-0,05$ & $-2,57$ & 5,33 \\
Carnaúba industrializada & $-3,23$ & $-0,46$ & $-1,83$ & 3,77 \\
Carnaúba bruta & 2,76 & $-1,77$ & $-6,54$ & 7,75 \\
Óleo de linhaça & $-7,86$ & 0,93 & 3,15 & 8,51 \\
\hline
\end{tabular}

$\Delta \mathrm{L}^{*}$ = variação na luminosidade; $\Delta \mathrm{a}^{*}=$ variação na matiz vermelha; $\Delta \mathrm{b}^{*}=$ variação na matiz amarela; $\Delta \mathrm{E}=$ variação na cor.

De acordo com Nzokou; Kamdem (2006), quanto menor o valor de $\Delta \mathrm{E}$, menor a variação da cor, sendo que a mudança passa a ser notável para os olhos humanos apenas em valores superiores a 1,5. Os mesmos autores classificam ainda variações que apresentam valores entre 3 e 6 como consideráveis e acima de 6 como altas.

Todos os tratamentos apresentaram mudanças perceptíveis. As aplicações de cera de Carnaúba industrializada e de verniz acarretaram em mudança mais sutil na cor original da madeira. Já o óleo de linhaça e cera de Carnaúba bruta produziram mudanças consideradas altas.

Em relação ao parâmetro $\mathrm{L}^{*}$, pode-se observar que o quando verniz e do óleo de linhaça foram usados, houve uma maior variação negativa, que pode ser interpretada como um escurecimento da madeira. No caso do óleo de linhaça também houve uma alteração positiva no parâmetro a*, que indica um realce da tonalidade vermelha. Esta combinação de fatores levou a uma mudança da percepção da cor da tonalidade rosa claro para uma tonalidade mais avermelhada. No caso do verniz, o efeito na mudança de cor foi semelhante, mas principalmente pela combinação entre a diminuição do parâmetro $b^{*}$ (pigmentação amarela) e a pouca variação o parâmetro $a^{*}$.

A Tabela 3 apresenta os valores médios dos parâmetros colorimétricos das madeiras tratadas com os diferentes produtos de acabamento durante o período de exposição ao intemperismo natural. Também é apresentado na Tabela 2 o efeito da aplicação dos produtos de acabamentos na cor $(\Delta \mathrm{E})$ em comparação com a cor da madeira sem tratamento.

Independente do uso e do tipo de produto de acabamento aplicado, o envelhecimento tende a escurecer a madeira de Maçaranduba (redução do parâmetro $L^{*}$ ), com exceção das peças tratadas com óleo de linhaça, que apresentaram valores de $L^{*}$ crescentes ao final dos quatro meses de exposição.

Em relação à formação da cor, houve uma tendência da coloração vermelha se acentuar ao longo do envelhecimento (aumento do parâmetro a*). Porém, em peças envernizadas, não houve diferença significativa entre os valores médios de a* ao longo do período de exposição, o que indicou uma proteção da coloração inicial. As maiores variações do parâmetro a* foram observadas após o primeiro mês do teste em peças tratadas com ceras de Carnaúba. Na prática, o efeito esbranquiçado causado após aplicação da cera foi perdido no primeiro mês de exposição.

A aplicação de verniz também foi capaz de manter a saturação (C) e tonalidade $\left(\mathrm{h}^{*}\right)$ das cores das madeiras expostas ao intemperismo. Os demais produtos tenderam a saturar a cor original logo após o primeiro mês de envelhecimento.

O comportamento da variação total da cor $(\Delta \mathrm{E})$ para os corpos de prova tratados com os diferentes produtos de acabamento durante os quatro meses de exposição às intempéries foi apresentado na Figura 1.

O efeito positivo do verniz na estabilização da cor pode ser observado no baixo $\Delta \mathrm{E}$. Por outro lado, a variação máxima ocorreu nas amostras tratadas com ceras de Carnaúba, tanto industrializada quanto bruta.

\section{DISCUSSÃO}

4.1. A cor da madeira de maçaranduba comercializada no Nordeste

Não houve diferença estatística entre os parâmetros colorimétricos de peças de maçaranduba comercializadas em diferentes estados da Região Nordeste. De acordo com a tabela de cores para madeira sugerida por Camargos; Gonzales (2001), as peças comercializadas apresentaram parâmetros próximos de madeiras classificadas como "rosa-acinzendato". Esta classificação se difere da encontrada por Silva et al. (2007) que encontraram valores mais baixos de $L^{*}, a^{*}$ e b* para discos de Manilkara huberi (Ducke) Chavalier retirados da base e classificaram a madeira como "marrom- 
avermelhado-escuro". Os valores encontrados para estes parâmetros também foram mais altos no trabalho de Ribeiro (2017) que classificou a madeira da espécie como "marromavermelhada". Porém o tom acinzentado da madeira já havia sido reportado por Vetter (1988) ao classificar a madeira de Manilkara huberi como "marrom acinzentado".

Os parâmetros $a^{*}$ e $b^{*}$, que determinam a tonalidade avermelhada da madeira, são influenciados pela quantidade de extrativos totais presentes nas madeiras, enquanto que tonalidades mais escuras (baixos valores de $\mathrm{L}^{*}$ ) são associadas a altos teores de componentes fenólicos (MOYA et al., 2012). Castro et al. (2017) avaliaram a composição química de oito espécies amazônicas e observaram que a madeira de Manilkara amazonica apresentou um teor de extrativos totais de $16,22 \%$, valor alto em comparação com as demais madeiras estudadas.

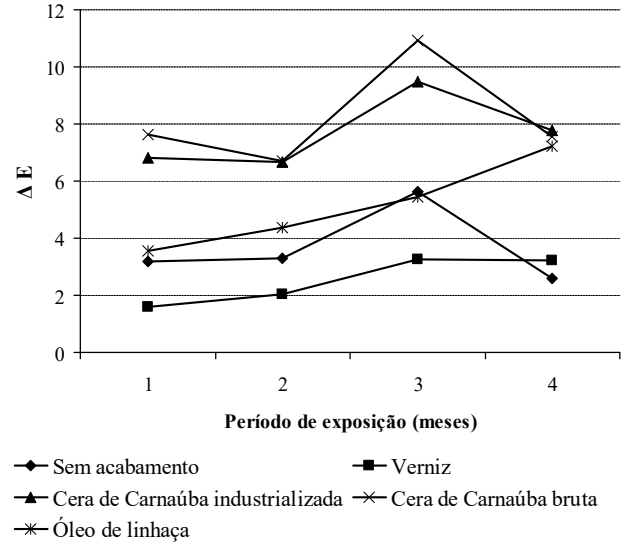

Figura 1. Variação do parâmetro $\Delta \mathrm{E}$ provocado pelo intemperismo natural.

Figure 1. Variation of $\Delta \mathrm{E}$ caused by natural weathering.

Tabela 3. Parâmetros colorimétricos de peças de maçaranduba tratadas com produtos de acabamento durante período de envelhecimento natural.

Table 3. Colorimetric parameters of Massaranduba wood treated with finishing products during natural weathering period.

\begin{tabular}{|c|c|c|c|c|c|c|}
\hline Tratamento & $\begin{array}{c}\text { Tempo de } \\
\text { exposição (meses) }\end{array}$ & $\mathrm{L}^{*}$ & $a^{*}$ & $b^{*}$ & $\mathrm{C}$ & $\mathrm{h}^{*}$ \\
\hline \multirow{5}{*}{ Sem acabamento } & 0 & $39,43 \mathrm{~d}$ & $9,30 \mathrm{a}$ & $13,24 \mathrm{a}$ & 16,19 a & $54,89 \mathrm{~d}$ \\
\hline & 1 & $37,67 \mathrm{bc}$ & $10,99 \mathrm{~b}$ & $15,31 \mathrm{c}$ & $18,85 \mathrm{~b}$ & $54,32 \mathrm{~d}$ \\
\hline & 2 & $37,18 \mathrm{~b}$ & $11,43 \mathrm{~b}$ & $14,40 \mathrm{~b}$ & $18,39 \mathrm{~b}$ & $51,57 \mathrm{~b}$ \\
\hline & 3 & 35,09 a & $12,66 \mathrm{c}$ & $14,51 \mathrm{~b}$ & $19,26 \mathrm{~b}$ & $48,88 \mathrm{a}$ \\
\hline & 4 & $37,99 \mathrm{c}$ & $10,86 \mathrm{~b}$ & $14,71 b c$ & $18,29 \mathrm{~b}$ & $53,54 \mathrm{c}$ \\
\hline \multirow{5}{*}{$\begin{array}{c}\text { Verniz } \\
\text { poliuretano }\end{array}$} & 0 & $34,04 \mathrm{c}$ & $12,83 \mathrm{a}$ & $7,30 \mathrm{a}$ & $14,76 \mathrm{a}$ & $29,60 \mathrm{~b}$ \\
\hline & 1 & $32,48 \mathrm{~b}$ & $12,93 \mathrm{a}$ & $7,05 \mathrm{a}$ & $14,73 \mathrm{a}$ & $28,55 \mathrm{ab}$ \\
\hline & 2 & $32,11 \mathrm{~b}$ & $12,57 \mathrm{a}$ & $6,66 \mathrm{a}$ & $14,23 \mathrm{a}$ & $27,82 \mathrm{a}$ \\
\hline & 3 & 30,79 a & $12,89 \mathrm{a}$ & $6,93 \mathrm{a}$ & $14,64 \mathrm{a}$ & $28,18 \mathrm{a}$ \\
\hline & 4 & $30,83 \mathrm{a}$ & $12,64 \mathrm{a}$ & $6,97 \mathrm{a}$ & $14,45 \mathrm{a}$ & $28,87 \mathrm{ab}$ \\
\hline \multirow{5}{*}{$\begin{array}{c}\text { Cera de } \\
\text { Carnaúba } \\
\text { industrializada }\end{array}$} & 0 & $44,26 \mathrm{c}$ & $8,60 \mathrm{a}$ & $11,37 \mathrm{a}$ & $14,26 \mathrm{a}$ & $52,79 \mathrm{~b}$ \\
\hline & 1 & $40,28 \mathrm{ab}$ & $11,46 \mathrm{~b}$ & $16,08 \mathrm{~b}$ & $19,75 \mathrm{~b}$ & $54,44 \mathrm{c}$ \\
\hline & 2 & $40,46 \mathrm{ab}$ & $11,81 \mathrm{~b}$ & $15,83 \mathrm{~b}$ & $19,76 \mathrm{~b}$ & $53,19 \mathrm{~b}$ \\
\hline & 3 & $38,00 \mathrm{a}$ & $13,45 \mathrm{c}$ & $16,59 \mathrm{bc}$ & $21,37 \mathrm{c}$ & $50,84 \mathrm{a}$ \\
\hline & 4 & $40,95 \mathrm{~b}$ & $11,83 \mathrm{~b}$ & $17,62 \mathrm{c}$ & $21,23 \mathrm{bc}$ & $56,03 \mathrm{~d}$ \\
\hline \multirow{5}{*}{$\begin{array}{c}\text { Cera de } \\
\text { Carnaúba bruta }\end{array}$} & 0 & $43,74 \mathrm{c}$ & $6,90 \mathrm{a}$ & $8,74 \mathrm{a}$ & $11,14 \mathrm{a}$ & $51,70 \mathrm{~b}$ \\
\hline & 1 & $38,38 \mathrm{ab}$ & $10,00 \mathrm{~b}$ & $13,20 \mathrm{~b}$ & $16,56 \mathrm{~b}$ & $52,85 \mathrm{c}$ \\
\hline & 2 & $39,56 \mathrm{~b}$ & $10,10 \mathrm{~b}$ & $12,89 \mathrm{~b}$ & $16,38 \mathrm{~b}$ & $51,91 \mathrm{~b}$ \\
\hline & 3 & $35,50 \mathrm{a}$ & $11,62 \mathrm{c}$ & $14,15 \mathrm{c}$ & $18,31 \mathrm{c}$ & $50,59 \mathrm{a}$ \\
\hline & 4 & $41,33 \mathrm{bc}$ & $10,15 \mathrm{~b}$ & $15,10 \mathrm{~d}$ & $18,20 \mathrm{c}$ & $56,09 \mathrm{~d}$ \\
\hline \multirow{5}{*}{ Óleo de linhaça } & 0 & $38,74 \mathrm{a}$ & $10,92 \mathrm{a}$ & $13,48 \mathrm{a}$ & $17,35 \mathrm{a}$ & $50,97 \mathrm{a}$ \\
\hline & 1 & $39,83 \mathrm{~b}$ & $12,42 \mathrm{c}$ & $16,50 \mathrm{~b}$ & $20,65 \mathrm{~b}$ & $53,01 \mathrm{c}$ \\
\hline & 2 & $40,46 \mathrm{c}$ & $12,60 \mathrm{c}$ & $17,12 \mathrm{c}$ & $21,26 \mathrm{~b}$ & $53,62 \mathrm{c}$ \\
\hline & 3 & $39,15 \mathrm{a}$ & $13,94 \mathrm{~d}$ & $18,01 \mathrm{~d}$ & $22,78 \mathrm{c}$ & $52,23 \mathrm{~b}$ \\
\hline & 4 & $43,65 \mathrm{~d}$ & $11,98 \mathrm{~b}$ & $18,68 \mathrm{e}$ & $22,19 \mathrm{c}$ & $57,31 \mathrm{~d}$ \\
\hline
\end{tabular}

$\mathrm{L}^{*}=$ luminosidade; $\mathrm{a}^{*}=$ matiz vermelha; $\mathrm{b}^{*}=$ matiz amarela; $\mathrm{C}=$ saturação; $\mathrm{h}^{*}=$ ângulo de tinta; Valores entre parênteses referentes ao coeficiente de variação; Médias seguidas por uma mesma letra, em cada coluna e dentro de um mesmo tratamento, não diferem estatisticamente (Tukey, $\mathrm{p}>0,05$ ).

\subsection{Aderência dos produtos de acabamento}

Os valores de aderência para o verniz poliuretano foi semelhante ao encontrado por Souza et al. (2011). Contudo os mesmos autores encontraram alta porcentagem de área descascada para o produto cera de Carnaúba (acima de 65\%), comportamento diferente ao observado neste estudo. Quando aplicado em peças de maçaranduba, as películas de ceras de Carnaúba, tanto a industrializada quanto a bruta, apresentaram valores de adesão satisfatórios.

\subsection{Efeito dos produtos de acabamento na cor.}

O efeito visível na cor com a aplicação do óleo de linhaça também foi relatado por outros autores. Liu et al. (2015) estudaram o efeito da aplicação deste produto na madeira chinesa Juglans mandshurica Maxim. e observaram um $\Delta \mathrm{E}$ de aproximadamente 9. Petric et al. (2004) relataram um $\Delta \mathrm{E}$ ainda mais alto $(15,2)$ para madeira de Pronus avium L tratada com óleo de linhaça.

$\mathrm{O}$ uso de cera de Carnaúba também foi relatado na literatura como quase transparente, sendo a variação da cor $\Delta \mathrm{E}$ $=1,95$ no caso da aplicação de camadas de cera com nanopartículas de óxido de zinco $(\mathrm{ZnO})$ em madeira de Picea abies (LOZHECHNIKOVA et al, 2017). No caso da madeira de Maçaranduba, a cera afetou a negativamente a tonalidade avermelhada da madeira, principalmente quando se utilizou a cera bruta sem conservantes, que também afetou negativamente a tonalidade amarela $\left(b^{*}\right)$ e resultou em peças esbranquiçadas. A diminuição da luz refletida em madeiras após aplicação de verniz também foi observada em madeiras claras de Couratari sp. (SILVA et al, 2007). 


\subsection{Envelhecimento natural}

Silva et al. (2007) também observaram uma variação na cor da Maçaranduba envernizada abaixo da sem acabamento em envelhecimento artificial. Por outro lado, a variação da cor em amostras tratadas com ceras de Carnaúba, apresentaram um resultado que se difere do apresentado por Lozhechnikova et al. (2017) que relataram uma redução do $\Delta \mathrm{E}$ de 17,6 para 10,6 quando a madeira de Picea abies envelhecida artificialmente foi tratada com cera de Carnaúba. Porém os mesmo autores observaram que a película da cera começou a sofrer degradação após 10 dias de irradiação de luz ultravioleta, desaparecendo quase que completamente.

Madeiras sem acabamento ou tratadas com ceras de Carnaúba apresentaram uma tendência de diminuição do $\Delta \mathrm{E}$ entre os meses 3 e 4. De acordo com Nzokou; Kamdem (2006), este padrão também foi observado em testes de envelhecimento artificial, e tende a ser seguido por um novo aumento de variação, de acordo com o embranquecimento da madeira exposta.

\section{CONCLUSÕES}

A madeira de maçaranduba na região Nordeste é esteticamente semelhante independentemente do local onde foi comercializada. Sua tonalidade avermelhada pode ainda ser realçada com uso de óleo de linhaça. Porém, a exposição da madeira à radiação solar, logo após o período de um mês, tem efeito significativo e compromete a cor original, mesmo com a aplicação de produtos naturais. Quando estes produtos são aplicados em madeira de uso externo, reaplicações mensais (no caso do óleo de linhaça) ou a cada dois meses (para cera de Carnaúba, independentemente de ser industrializada ou bruta diluída em querosene) são recomendadas. Já o verniz poliuretano apresentou maior vida útil na proteção da cor após exposição.

\section{REFERÊNCIAS}

ABNT_ASSOCIAÇÃO BRASILEIRA DE NORMAS TECNICAS. NBR 11003: Tintas - Determinação da aderência. Rio de Janeiro; 2009. 13 p.

ATAYDE. C. M; GONÇALVEZ. J. C; CAMARGOS. J. A. Características colorimétricas entre as seções anatômicas da madeira de muirapiranga (Brosimum sp.). Cerne, Lavras, v. 17, n. 2, p. 231-235, abr./jun. 2011. DOI: http://dx.doi.org/10.1590/S0104-77602011000200011

BAYSAL, E.; TOMAK, E. D.; OZBEY, M.; ALTIN, E. Surface properties of impregnated and varnished Scots pine wood after accelerated weathering. Coloration Technology, West Yorkshire, v. 130, n. 2, p. 140-146, dez. 2013. DOI: http://dx.doi.org/10.1111/cote. 12070

BULIAN, F.; GRAYSTONE, J. A. Wood coatings: theory and practice. Amsterdam: Elsevier, 2009. 320 p.

CAMARGOS, J. A.; GONÇALEZ, J. C. A Colorimetria aplicada como instrumento na elaboração de uma tabela de cores de madeira. Brasil Florestal, Brasília, v. 71, p. 3041, set. 2001.

CASTRO, V. G.; AZAMBUJA, R. R.; BILA, N. F.; PARCHEN, C. F. A.; SASSAKI, G. L.; IWAKIRI, S. Correlation between chemical composition of tropical hardwoods and wood-cement compatibility. Journal of Wood Chemistry and Technology, Londres, v. 37, n. 1, p. 28-34, 2017.2 DOI: https://dx.doi.org/10.1080/02773813.2017.1355390
CHANG, C. W.; LU, K. T. Linseed-oil-based waterborne UV/air dual-cured wood coating. Progress in Organic Coating, v. 76, n. 7-8, p. 1024-1031, jul./ago. 2013. DOI: https://dx.doi.org/10.1016/j.porgcoat.2013.02.020

EN EUROPEAN STANDARD. EN 927-3: Paints and varnishes - Coating materials and coating systems for exterior wood - Part 3: Natural weathering test. 2000.

FIEDLER, N. C.; COSTA, A. F.; SOARES, T. S.; LEITE, A. M. P. Caracterização do segmento de madeira serrada em três municípios do estado do Pará. Revista Brasileira de Ciências Agrárias, Recife, v. 7, n. 1, p. 111-116, jan./mar. 2012. DOI: http://dx.doi.org/10.5039/agraria.v7ila985

GONÇALEZ. J. C; FÉLIX. T. L. F; GOUVEIA. F. N; CAMARGOS. J. A. A; RIBEIRO.P. G. Efeito da radiação ultravioleta na cor da madeira de freijó (Cordia goeldiana Huber) após receber produtos de acabamentos. Ciência Florestal, Santa Maria, v. 20, n. 4, p. 657-664, 2010. DOI: http://dx.doi.org/10.5902/198050982424

LIU, X, Y.; TIMAR, M. C.; VARODI, A. M.; YI, S. L. Tung oil and linseed as traditional finishing materials important for furniture conservation. Proligno, Brasov, v. 11, n. 4, p. 571-579. 2015.

LOZHECHNIKOVA, A.; BELLANGER, H.; MICHEN, B.; BURGERT, I.; OSTERBERG, M. Surfactant-free carnauba wax dispersion and its use for layer-by-layer assembled protective surface coatings on wood. Applied Surface Science, Atlanta, v. 396, p. 1273-1281. 2017. DOI: http://dx.doi.org/10.1016/j.apsusc.2016.11.132

MOYA, R.; FALLAS, R. S.; BONILLA, P. J.; TENORIO, C. Relationshio between wood color parameters measured by the CIELab system and extractive and phenol content in Acacia mangium and Vochysia guatemalensis from fastgrowth plantations. Molecules, Basel, v. 17, n. 4, p. 36393652.2012 .2 DOI: https://dx.doi.org/10.3390/molecules 17043639

NZOKOU, P.; KAMDEM, D. P. Influence of wood extractives on the photo-discoloration of wood surfaces exposed to artificial weathering. Color Research and Application, Medford, v. 31, n. 5, p. 425-434, out. 2006. DOI: http://dx.doi.org/10.1002/col.20248

PETRIC, M.; KRICEJ, B.; HUMAR, M.; PAVLIC, M.; TOMAZIC, M. Patination of cherry wood and spruce wood with ethanolamine and surface finishes. Surface Coating International Part B: Coating Transactions, New York, v. 87, n. 3, p. 149-156, set. 2004. DOI: https://dx.doi.org/10.1007/BF02699635

QUEIROZ. F. L. C; GONÇALEZ. J. C; MENEZZI. C. H. D; RIBEIRO. E. S; LIMA. C. M. Intemperismo artificial em lâminas de Tectona grandis tratadas com produtos de acabamento. Floresta e Ambiente, Seropédica, v. 23, n. 4, p. 573-581, mar. 2016. DOI: http://dx.doi.org/10.1590/2179-8087.126315

RIBEIRO, E. S. Propriedades tecnológicas de vinte espécies de madeiras tropicais comercializadas pelo estado de Mato Grosso. 2017. 210f. Tese (Doutorado em Ciências Florestais) - Universidade de Brasília, Brasília, 2017.

ROSA, R. A.; FRANÇA, L. C. A.; SEGUNDINHO, P. G. A.; LUBE, V. M.; PAES, J. B. Caracterização da madeira de maçaranduba (Manilkara sp.) por métodos destrutivos e não destrutivos. Ciência da Madeira, Pelotas, v. 5, n. 1, p. 68-78, mai. 2014. DOI: http://dx.doi.org/10.12953/2177-6830.v05n01a07 
SANTANA, A. C.; SANTOS, M. A. S.; OLIVEIRA, C. M. Comportamento histórico da produção e comércio de madeira do estado do Pará nos mercados local e internacional. Amazônia Ciência \& Desenvolvimento, Belém, v. 6, n. 11, p. 63-90, jul./dez. 2010.

SANTOS J. A; DUARTE C. Degradação e proteção superficial da madeira em exterior. Corrosão e Protecção de Materiais, Lisboa, v. 32, n. 1, p. 10-18, mar. 2013.

SILVA. J. O; PASTORE. T. C. M; PASTORE. F. J. Resistência ao intemperismo artificial de cinco madeiras tropicais e de dois produtos de acabamento. Ciência Florestal, Santa Maria, v. 17, n. 1, p. 17-23, jan./mar. 2007. DOI: http://dx.doi.org/10.5902/198050981931

SILVA. E. S; STANGERLIN. D. M; GATTO. D. A, CALEGARI. L; PARIZ. E. Colorimetria da madeira de oito espécies nativas do estado do Rio Grande do Sul, Brasil. Ciência da Madeira, Santa Maria, v. 6, n. 1, p. 3137, abr. 2015. DOI: http://dx.doi.org/10.12953/21776830/rcm.v6n1p31-37

SOUZA. M. O. A; SILVA. J. C; EVANGELISTA. W. V. Aplicação de acabamentos superficiais em madeira de Eucalyptus camaldulensis Dehnh. e Eucalyptus urophylla S.T. Blake visando ao uso na indústria moveleira. Scientia Forestalis, Piracicaba, v. 39, n. 92, p. 403-409, dez. 2011.

VETTER, R. E. Colorimetria aplicada em pesquisa de madeira. Acta Amazonica, Manaus, v. 18, n. 1-2, p. 179188, mar./jun. 1988. DOI: http://dx.doi.org/10.1590/180943921988182188 